\title{
Intervenciones de enfermería en neonatos con presión positiva continua*
}

\author{
Nursing Intervention in Neonates with Continuous Positive Pressure \\ Intervenções de enfermagem em neonatos com pressão positiva contínua
}

Cleotilde García Reza ${ }^{a}$

DOI: https://doi.org/10.11144/Javeriana.ie20-1.ienp

Universidad Autónoma del Estado de México, México

cgarc0506@yahoo.com.mx

Maria Andrea Mejía-Flores

Secretaría de Salud del Estado de México, México

Fecha de recepción: 16 Diciembre 2015

Fecha de aprobación: 05 Agosto 2016

\author{
Liliana Guadarrama Pérez \\ Secretaría de Salud del Estado de México, México \\ Vicenta Gómez Martínez \\ Universidad Autónoma del Estado de México, México
}

\section{Resumen:}

Objetivo: describir las intervenciones de enfermería en neonatos con presión positiva continua con prongs, hospitalizados en una unidad neonatal mexicana. Métodos: corte transversal en una muestra no aleatoria por conveniencia, conformada por veinticinco enfermeras que tenían bajo sus cuidados a recién nacidos con sistema de presión positiva con prongs. La recolección de datos fue realizada con un cuestionario autoplicado. El análisis fue descriptivo, y se obtuvieron medidas de tendencia central de las variables cuantitativas que mostraron una distribución normal. Las variables cualitativas se describen con valores relativos. Se respetaron aspectos éticos según la reglamentación vigente mexicana. Resultados: participaron veinticinco enfermeras con grado académico: el $88 \%$ con licenciatura y solo el $24 \%$ especialistas neonatólogas. Los neonatos usan la presión positiva continua: el $52 \%$ de 3 a 5 días; el 20\% de 6 a 7 días y el 28\% de 8 a 12 días. El nivel de lesión nasal y los resultados con prongs, mediante el cuidado constante de la enfermera sobre la aparición de estas, evidenciaron que el 60\% de los neonatos presentó lesión leve; el 36\%, moderada, y el $4 \%$, severa. Conclusiones: las intervenciones de cuidado del neonato deben dirigirse a minimizar las lesiones de piel y tabique nasal para contribuir con las normas internacionales de seguridad del neonato.

Palabras clave: recién nacido, presión positiva, enfermería neonatal, cuidado, nariz.

\section{Abstract:}

Objective: To describe nursing interventions in neonates with continuous positive airway pressure with prongs, Mexican hospitalized in neonatal unit. Methods: Cross-section in a nonrandom convenience sample, composed of 25 nurses who were under their care to newborns, positive pressure system with prongs. Data collection was performed with self-administered survey. Data analysis was descriptive, measures of central tendency for quantitative variables that showed a normal distribution were obtained. The qualitative variables are described in relative values. Ethical aspects were respected as required by regulations. Results: 25 nurses participated degree: $88 \%$ graduate and only $24 \%$ neonatologist specialists, Infants use continuous positive airway pressure: $52 \%$ of 3-5 days $20 \%$ of 6-7 days and $28 \%$ of 8-12 days with respect to the level of nasal lesions, results with prongs, through constant care nurse about the appearance of these, $60 \%$ of neonates presented mild injury, $36 \%$ moderate and $4 \%$ severe. Conclusions: It requires nursing care interventions careful of the newborn to minimize skin lesions and nasal septum and contribute to international safety standards neonate.

Keywords: infant newborn, positive pressure, care, neonatal nursing, nose.

\section{Resumo:}

Objetivo: describir as intervenções de enfermagem em neonatos com pressão positiva contínua com prongs, internados em uma unidade neonatal mexicana. Métodos: de corte transversal em uma amostra por conveniência não aleatória, composta por 25 enfermeiros que dão cuidados ao recém-nascido com sistema de pressão positiva com prongs. A coleta de dados foi realizada

Notas de autor 
com um questionário autoaplicável. A análise dos dados foi descritiva, foram obtidas medidas de tendência central para variáveis quantitativas que apresentaram distribuição normal. As variáveis qualitativas são descritos em valores relativos. Aspectos éticos foram respeitados conforme exigidos pelos regulamentos mexicanos. Resultados: 25 enfermeiros com grau académico: $88 \%$ licenciatura e apenas $24 \%$ neonatologas especialistas. Os neonatos com o uso da pressão positiva contínua: $52 \%$ de 3-5 dias, o $20 \%$ de 6-7 dias e o $28 \%$ de 8-12 dias, com respeito ao nível de lesões nasais, os resultados com prongs, o 60\% de neonatos apresentou lesão leve, $36 \%$ moderada e $4 \%$ grave. Conclusões: as intervenções de cuidado devem ser encaminhadas a minimizar lesões de pele e septo nasal e contribuir com as normas de segurança internacionais do neonato.

Palavras-chave: recém-nascido, pressão positiva, cuidado, enfermagem nonatal, nariz.

\section{Introducción}

En este tercer milenio, el cuidado de enfermería vive una serie de transformaciones respecto a la tecnología de ventilación con presión positiva continua en vías aéreas (CPAP, por su sigla en inglés) [1]. Constituye una opción terapéutica para recién nacidos prematuros con síndrome de dificultad respiratoria, con el uso de dispositivo nasal prongs, en su forma más simple y menos invasivo de suministrar [2].

En los recién nacidos, el uso de pretérmino es cada vez más frecuente [3]; por ello, es necesario que enfermería dirija las intervenciones de cuidado a este tipo de dispositivo nasal prongs [4]. Esta es una intervención que incrementa la efectividad del cuidado para generar conocimientos desde su perspectiva práctica disciplinar, apoyada en la relación dialógica entre el profesional de enfermería y el neonato [3,5].

Estas intervenciones se basan en el conocimiento y juicio clínico para favorecer la vida del neonato con este sistema de ventilación; de ahí que sea imprescindible vigilar de manera permanente tres factores: el flujo continuo de la mezcla de gases (aire comprimido y oxígeno) calentado y humidificado; el dispositivo de conexión del sistema a las vías aéreas del neonato, como máscaras faciales, prongs nasales, cánulas nasofaríngeas o endotraqueales, y vigilar la protección cutánea [6].

El uso prolongado de prongs en la unidad de cuidados neonatales puede causar lesiones nasales en neonatos, desde una simple hiperemia de la mucosa nasal, pasando por sangrado, formación de costras, necrosis, hasta la destrucción total del tabique anterior; por tanto, cuidados oportunos pueden reducir las cifras iatrogénicas. La Organización Mundial de la Salud [7] afirma que las intervenciones deben resolver problemas relacionados con la seguridad del paciente, pues las consideran un nuevo recurso para ayudar a los hospitales de tercer nivel a evitar muertes, así como lesiones prevenibles que salven vidas [6,8].

El profesional de enfermería deberá asumir un nuevo paradigma en su función de cuidador, a fin de identificar respuestas humanas, clasificarlas y priorizarlas, y, con ello, planificar u organizar los cuidados de neonatos. Estas intervenciones se deberán caracterizar por su calidad científica y humanización del cuidado profesional [5,9]; por tal razón, redefinir en teoría y práctica la dimensión de los cuidados constituye un escenario para desarrollar de manera esencial el cuidado de enfermería, en un proceso de interacción dirigido a cuidar, apoyar y acompañar en la recuperación de la salud mediante la identificación de las necesidades humanas del neonato $[8,10]$.

En los últimos años, el profesional de enfermería ha transformado sus cuidados centrados en los neonatos como seres humanos [11], no como un cuerpo enfermo con patología pulmonar neonatal; las intervenciones a estos neonatos se orientan a proporcionar cuidados relacionados con el confort, cuidados de la piel, limpieza nasal y cambios de posición, con el fin de aumentar calidad del cuidado de enfermería.

Por lo tanto, es importante que la enfermera que está al cuidado del neonato posea habilidades para describir, interpretar y hallar soluciones a nuevos problemas para valorar al neonato en todo su contexto de enfermedad [9,10].

En México existen pocos estudios relacionados con el cuidado de enfermería asociados al uso de CPAP con prongs. Así surgió el interés de la presente investigación, que se basa en describir las intervenciones de enfermería durante la instalación, el manejo y el mantenimiento en la aplicación de CPAP en las vías 
aéreas con prongs, y ya que las actividades de enfermería inducen a la interacción directa con el recién nacido en su capacidad residual funcional del área de superficie alveolar para el intercambio de gases, nos planteamos el siguiente objetivo: describir las intervenciones de enfermería en neonatos con CPAP con prongs, hospitalizados en una unidad neonatal mexicana.

\section{Material y métodos}

El enfoque de la investigación es cuantitativo de corte transversal, realizado en la unidad de cuidados intensivos neonatales (UCIN) de un hospital de alta especialidad en el área perinatal para población abierta del Estado de México, que proporciona atención obstétrica y neonatal de alto riesgo. Cuenta con 72 camas censables y 73 no censables. Tiene una población de 332 enfermeras distribuidas en los diferentes turnos y en los servicios de alojamiento conjunto y transición, cuidados intensivos e intermedios neonatales, cuidados intensivos obstétricos, reanimación neonatal, estimulación temprana, cardiología neonatal genética, neurología, neurocirugía, entre otros. La UCIN cuenta con 28 camas no censables. El índice de mortalidad incluye: prematurez, síndrome de dificultad respiratoria, cardiopatía congénita, sepsis neonatal, malformaciones congénitas, síndrome de mala adaptación pulmonar y neumonía congénita.

La muestra se seleccionó conveniencia y estuvo conformada por veinticinco enfermeras que realizaban intervenciones dirigidas a recién nacidos mediante CPAP nasal con prongs, hospitalizados en la UCIN. Los criterios de inclusión fueron la permanencia durante más de un año en el servicio de neonatos y tener a su cuidado recién nacidos pretérmino con CPAP nasal con prongs.

El levantamiento de datos se realizó mediante un cuestionario autoplicado de dos apartados: en el primero, se valoraron aspectos generales del recién nacido, como edad gestacional por Capurro al momento del nacimiento, edad, peso actual, sexo, patología(s) diagnosticada(s) al momento de realizar el estudio, días de estancia hospitalaria, días de uso de CPAP; en el segundo, dirigido a las enfermeras, las preguntas versaron sobre datos sociodemográficos, intervenciones de enfermería acerca de cuatro aspectos básicos de la aplicación de la terapia: 1) circuito y humidificador, 2) interfaz, 3) posición y 4) monitoreo. Para eso, se aplicó una prueba piloto en seis enfermeras, durante la guardia nocturna C. Con los resultados se reformularon tres preguntas, para después aplicar la versión final.

Para confirmar los datos se utilizó la observación participante. Se verificaron a través del contacto directo del investigador con el fenómeno de estudio en sus propios contextos de trabajo: desde los registros de enfermería durante las intervenciones a veinticinco prematuros en la valoración nasal de la unidad neonatal de un hospital de tercer nivel, relacionado con el circuito y humidificador, funcionalidad, temperatura, nivel de agua y condiciones del circuito; en el aspecto de interfaz, tamaño de prongs utilizados, posición del neonato, tamaño del gorro y tensores de sujeción; en cuanto a la postura, los aspectos relacionados con la posición del neonato. Al monitorearlo, se observaron intervenciones de higiene, lubricación, condiciones de la nariz, aspiración de secreciones. La aparición de lesiones en las notas de enfermería se registró en los diferentes turnos - matutino, vespertino, nocturno ( $\mathrm{A}, \mathrm{B}$ y C ) y especial diurno- L La observación se realizó durante la instalación y colocación del equipo prongs, así como los cuidados de enfermería a los recién nacidos pretérmino durante este procedimiento.

Posterior a la observación, se procedió a comparar la información con el expediente y las notas de enfermería del recién nacido pretérmino, donde fueron identificados datos generales como: días de estancia hospitalaria en la UCIN, días de uso de CPAP, accesorio para su aplicación, grado de lesión, perfil académico del profesional de enfermería y turno.

Los datos fueron recolectados por la investigadora principal, en el periodo julio a noviembre del 2014. Se contactó a las responsables del cuidado de los neonatos, quienes fueron invitadas a participar voluntariamente del estudio, con la firma del consentimiento informado, lo que permitió recoger los datos incluidos en la investigación. 
Dentro de las consideraciones legales estuvo el Reglamento de la Ley General de Salud Mexicano en Materia de Investigación para la Salud (título segundo): de los aspectos éticos de la investigación en seres humanos. De este el capítulo I, artículo XIII, establece: "En toda investigación en la que el ser humano sea sujeto de estudio, deberán prevalecer el criterio del respeto a su dignidad y la protección de sus derechos y bienestar" [12]. También se tuvo en cuenta la Resolución 574/2013 y el Comité de Bioética de dicha institución.

Una vez recolectados los datos, se almacenaron y tabularon en una planilla electrónica. El análisis descriptivo se realizó con el programa Statistical Package for the Social Sciences (SPSS) versión 21.0 del 2014, que presenta los resultados frecuencia y porcentajes en forma de tablas, para finalmente analizan las intervenciones de enfermería del recién nacido pretérmino con prongs de un hospital mexicano de alta especialidad.

\section{Resultados}

El perfil académico de los 25 profesionales en enfermería de un hospital de alta especialidad estuvo constituido por licenciadas en enfermería (64\%), por enfermeras generales (24\%) y por enfermeras especialistas en perinatología $(12 \%)$ (tabla 1$)$.

TABLA 1

Perfil académico de enfermería de un hospital de alta especialidad, México, 2014

\begin{tabular}{|l|r|r|}
\hline \multicolumn{1}{|c|}{ Variable } & Frecuencia & Porcentaje \\
\hline Licenciatura & 16 & 64 \\
\hline Enfermera general & 6 & 24 \\
\hline Enfermera especialista & 3 & 12 \\
\hline Total & 25 & 100 \\
\hline
\end{tabular}

Fuente: elaboración propia.

En cuanto a los recién nacidos prematuros que estaban al cuidado de las enfermeras en la UCIN, se observó la edad gestacional: el 68\% de ellos tenía de 27 a 33 semanas de gestación, y el 32\%, de 34 a 37 semanas, situación que predispone a los recién nacidos al desarrollo de lesiones nasales, aunado al no mantener su temperatura corporal y presentar menor grasa corporal y fragilidad de la piel (tabla 2).

TABLA 2

Edad gestacional del neonato con presión positiva continua de un hospital de alta especialidad, México, 2014

\begin{tabular}{|l|r|r|}
\hline Variable & Frecuencia & Porcentaje \\
\hline $27-30$ & 8 & 32 \\
\hline $31-33$ & 9 & 36 \\
\hline $34-37$ & 8 & 32 \\
\hline Total & 25 & 100 \\
\hline
\end{tabular}

Fuente: elaboración propia.

Respecto al diagnóstico más frecuente de los recién nacidos: el 32\% presentó síndrome de dificultad respiratoria modificada por surfactante; el $20 \%$, restricción del crecimiento intrauterino, y el 12\%, neumonía congénita e intrauterina. En cuanto a los días de uso de la CPAP: para el 52\% fue de 3 a 5 días; para el 20\%, 
de 6 a 7 días, y para el 28\%, de 8 a 12 días. Es evidente que en el neonato, después del octavo día en uso del CPAP, aumenta el riesgo de lesiones nasales (tabla 3).

TABLA 3

Días de uso en el neonato con presión positiva continua de un hospital de alta especialidad, México, 2014

\begin{tabular}{|l|r|r|}
\hline Variable & Frecuencia & Porcentaje \\
\hline $3-5$ & 13 & 52 \\
\hline $6-7$ & 5 & 20 \\
\hline $8-12$ & 7 & 28 \\
\hline Total & 25 & 100 \\
\hline
\end{tabular}

Fuente: elaboración propia.

Respecto a los resultados de la observación sobre las intervenciones de enfermería en la revisión del CPAP, el 76\% no revisan la funcionalidad al momento de la calibración; solo el 24\% lo lleva a cabo. En cuanto a las intervenciones de enfermería dirigidas al nivel de agua del humidificador, el 60\% vigila este parámetro y el $40 \%$ no lo hace. Es posible que esto aumente la gravedad y afecte el éxito del CPAP como método primario de asistencia ventilatoria del neonato (tabla 4 ).

TABLA 4

Intervenciones de enfermería del neonato con CPAP en la revisión y calibración, nivel de agua y humidificación, hospital de alta especialidad, México, 2014

\begin{tabular}{|l|r|r|}
\hline Variable & Frecuencia & Porcentaje \\
\hline \multicolumn{3}{|c|}{ Calibración } \\
\hline Sí & 19 & 76 \\
\hline No & 6 & 24 \\
\hline \multicolumn{3}{|c|}{ Nivel de agua del humidificador } \\
\hline Sí & 15 & 60 \\
\hline No & 10 & 40 \\
\hline \multicolumn{3}{|c|}{ Revisión de tubos } \\
\hline Sí & 20 & 80 \\
\hline No & 5 & 20 \\
\hline \multicolumn{3}{|c|}{ Vigila el tamaño de prongs } \\
\hline Sí & 21 & 84 \\
\hline No & 4 & 20 \\
\hline Total & 25 & 100 \\
\hline
\end{tabular}

Fuente: elaboración propia.

En este sentido, para la revisión de los tubos, el $80 \%$ de las enfermeras no vigila la posición de los tubos corrugados, acción que puede agravar las lesiones de la mucosa traqueal. Al vigilar el tamaño de prongs, el $84 \%$ verifica que sea adecuado al perímetro cefálico, lo cual favorece el uso del CPAP. En cambio, 16\% no lo lleva a cabo. Asimismo, una excesiva humidificación favorece la retención hídrica; por el contrario, si es insuficiente, condensa las secreciones y da lugar a la obstrucción de las vías aéreas (tabla 4).

Respecto a verificar el tensor para sujetar prongs, el $72 \%$ no lo hace. Durante la observación se constató que el 28\% de las enfermeras lo lleva a cabo, protegen la piel del recién nacido con Cavilon y cambian la posición del neonato con CPAP. En cuanto a la aparición o presencia de alguna lesión, el 52\% de las enfermeras lo valora; esto se manifiesta con la presencia de las lesiones nasales: un 60\% de los neonatos tuvo lesión leve; un $36 \%$, moderada, y un $4 \%$, severa. 


\section{Discusión}

La UCIN constituye un ambiente terapéutico apropiado para el tratamiento del neonato en estado grave. Su cuidado con CPAP se ha vuelto una tendencia en el mundo; sin embargo, también su uso disminuyó por un par de causas: la disponibilidad de ventiladores mecánicos diseñados específicamente para neonatos y altas tasas de fallas en neonatos menores de $1500 \mathrm{~g}$, que demandan más cuidados de enfermería [3,5].

La importancia de las intervenciones de enfermería radica en la formación profesional; las especialistas en neonatología poseen un perfil académico específico para asumir la responsabilidad en las intervenciones del recién nacido con CPAP. Son profesionales calificados en el cuidado del neonato en estado crítico; sin embargo, estos resultados muestran una separación entre la formación y su vínculo con avances e innovaciones, reflejo de las intervenciones y aportes enfermera-neonato, que se orienta bajo el marco del metaparadigma $[13,14]$.

En la profesión de enfermería es imprescindible ser lo suficientemente responsables al aplicar conocimientos y enfrentar el cuidado desde las respuestas humanas de una compleja red de posibilidades para un abordaje acorde con su formación [15]. Es fundamental incorporar profesionales calificados con vastos conocimientos científicos para hacer eficiente el cuidado, y debido al impulso de diferentes tecnologías, se requieren diferentes saberes, en busca de la reducción de la mortalidad neonatal. En pro de ello, es preciso proporcionar soluciones vertiginosas y exhaustivas desde diferentes perspectivas, como las que exponen los derechos sexuales y reproductivos, la bioética y los principios del autocuidado $[9,16,17]$.

Otro aspecto de los resultados fue la observación. Es evidente la falta de intervenciones de enfermería acordes con los requerimientos del neonato; de ahí la necesidad de sensibilizar la responsabilidad profesional y legal, con la firmeza de índole científico, para otorgar un cuidado profesional con un rigor altamente calificado $[15,18]$. El neonato, por su estado crítico, experimenta cambios agresivos fuera del ambiente uterino, pero decisivos para su supervivencia. Los neonatos con CPAP $[11,13]$ alteran su patrón respiratorio; de ahí que el profesional de enfermería, con sus intervenciones, deba mantener dicho patrón. Es responsable de la oxigenación y ventilación, pues a través de la permeabilidad de las vías aéreas, utiliza técnicas y procedimientos especialmente diseñados para la prevención de complicaciones respiratorias; por ello, es menester considerar el perfil académico del profesional de enfermería, para relacionar su preparación y pericia, a efectos de garantizar los cuidados básicos de protección e higiene para el mantenimiento de la vida del neonato con CPAP nasal $[10,17]$.

La intervención de enfermería en el cuidado del neonato con CPAP en las vías aéreas con prongs es un asunto poco referido. Los resultados de este estudio evidenciaron que a la UCIN ingresan recién nacidos pretérmino con inmadurez anatómica funcional del sistema respiratorio por inadaptación o síndrome de dificultad respiratoria. Su principal problema es el colapso alveolar durante la espiración a causa del déficit de surfactante o apnea por inmadurez. Entonces, la tendencia actual para su manejo es un tipo de ventilación no invasiva, la CPAP $[16,19]$, que mejora los cambios de gases y previene el colapso de las vías aéreas durante el esfuerzo inspiratorio. Así, la seguridad del neonato se centra en realizar intervenciones precisas, en el lugar correcto, donde es prioritario garantizar el cuidado del neonato y fortalecer las intervenciones de enfermería en el área de la UCIN $[7,20]$.

La intervención de enfermería hacia los neonatos se fundamenta en la identificación, el seguimiento y el control de los cuidados. Dichas intervenciones las regula y estructura la Clasificación de Intervenciones de Enfermería [21], órgano que vigila los parámetros del CPAP. Con frecuencia, estos coinciden o se ajustan a los programados. La enfermera, por tanto, registra los parámetros ventilatorios y verifica los valores gasométricos realizados, y así es como el cuidado de enfermería deja de ser una acción de rutina y se convierte en un actuar responsable al valorar, diagnosticar, expandir, diseminar, implementar y evaluar al neonato sobre una base de conocimientos de la ciencia de enfermería [18,22]. 
Al examinar las narinas del recién nacido, el estudio reveló que un alto porcentaje del personal de enfermería no realiza esta intervención, lo cual es alarmante, debido al incremento de lesiones y complicaciones [14,23]; además, esto disminuye la capacidad residual funcional de la superficie alveolar. Desde esta perspectiva, los neonatos están expuestos a procesos potenciadores de deterioros generados por el cuidado rutinario $[24,25,26]$. Por ello, el profesional de enfermería necesita revisar las intervenciones independientes, las de colaboración, así como los cuidados directos e indirectos en el neonato durante la pre y postaplicación del CPAP con prongs.

Se infiere, así, que los cuidados deben darse de forma cotidiana, de acuerdo con las necesidades del recién nacido con prongs, a fin de prevenir daños potenciales en el tabique nasal y lograr el bienestar del recién nacido. Otro aspecto identificado fue el no registro de los efectos de sus intervenciones, lo que hace imposible evaluar la naturaleza, la eficacia y la calidad de los cuidados de enfermería, al igual que su parcial identificación $[27,28]$.

Estos resultados muestran los cuidados brindados al neonato. Es indudable que no existen evidencias del juicio clínico de enfermería; además, el registro de estas intervenciones se limita a describirlas bajo un sistema rutinario. Las notas de enfermería, ya sean digitales o manuales [19,28], pocas veces pasan a formar parte del histórico del neonato. Ello podría transformar las intervenciones de imaginarias a intervenciones reales [17,27]. Así, ese registro puede garantizar el cuidado del neonato con CPAP.

La tendencia de utilizar el prongs por un menor tiempo posible previene complicaciones asociadas; pero su uso temprano disminuye la necesidad de ventilación mecánica y surfactante, produce mayor eficiencia ventilatoria y menor exposición a oxígeno. Mantener un cuidado especial sobre la posición del recién nacido evita el uso de accesorios para la oxigenación. Con el solo cambio de posición y rotando la cabeza de manera alterna, se mantiene permeable la vía aérea y disminuye la severidad de las lesiones del tabique nasal $[1,29]$.

Se evidencia que existe menor riesgo de desplazamiento de prongs con el uso de placas de hidrocoloide, el gel silicón $[9,20,29]$, así como el protector cutáneo, a fin de evitar la fricción directa de las prongs con la columela y el tabique. De esta manera, es visible el cuidado individual del neonato y cómo es un arte conjugar la ciencia con el sentido cotidiano del profesional de enfermería en beneficio del confort del neonato [19,24,28].

Mantener las fosas nasales limpias y lubricadas disminuye el grado de lesión [7,25,29]. Ese hecho deja claro que los asuntos relacionados con el costo-beneficio deberían ser reevaluados en centros de estudio que utilizan prongs; sin embargo, las dificultades observadas entorpecen el cuidado científico y lo convierten en desafío para encontrar concepciones actualizadas de la práctica que respondan a las intervenciones derivadas de los avances científicos y tecnológicos $[9,24,26]$. Es responsabilidad ética de las enfermeras planificar y evaluar las intervenciones de una manera coherente, a fin de garantizar la seguridad de neonato, el bienestar, confort y la continuidad del cuidado del neonato [25,28].

\section{Conclusiones}

Es evidente la necesidad de intensificar intervenciones que disminuyan la frecuencia y el grado de las lesiones que se lleguen a producir, ya que todos los neonatos de esta muestra evidenciaron lesiones desde leves hasta severas. Lo anterior en apoyo a las normas internacionales de seguridad del neonato, el cuidado, la supervisión, las habilidades para la protección de la piel y la observación de la estructura nasal, a fin de garantizar una terapia exitosa.

A partir de los resultados, las intervenciones de enfermería están relacionadas directamente con mantener el sistema y la vigilancia del CPAP. Las intervenciones son base del cuidado humano; por ello, se debe sensibilizar al profesional de enfermería sobre su continua capacitación, el uso de protocolos y la lista de cotejo, para fortalecer las debilidades y lograr un cuidado de calidad. 


\section{Limitaciones}

La muestra analizada fue reducida, y los resultados de la investigación fueron parciales. Por ello, no se pueden describir los beneficios o riesgos a largo plazo. Es necesario continuar con esta investigación en una muestra mayor para hacerla más precisa, y también contar con pruebas estadísticas que validen la sensibilidad del método.

\section{Referencias}

1. Santos RRR, Cardoso LMVL, Silva da FGR, Lúcio LIM. Aplicação de manual educativo sobre a pele do RN com estudantes de enfermagem. Rev Eletrônica Enfermagem [internet]. 2007;9(3):759-71. Disponible en: http://w ww.fen.ufg.br/revista/v9n3a

2. Domínguez DF. Ventilación de alta frecuencia en neonatología: a quiénes y cómo ventilar. Rev Cubana Pediatr [internet]. 2005 [citado 2013 mar 7];77(2). Disponible en: http://bvs.sld.cu/revistas/ped/vol77_2_05/ped04 205.htm

3. Covarrubias OL. Papel actual de la presión positiva continua en la vía aérea en el síndrome de la dificultad respiratoria, nueva evidencia. Bol Med Hosp Infant Mex [internet]. 2012 [citado 2013 abr 14];69(6):422-30. Disponible en: http://www.medigraphic.com/pdfs/bmhim/hi.pdf

4. Alves MA, Santos SDAE, Souza GT. Prevenção de lesões nasais secundárias ao uso de pressão positiva contínua nas vias aéreas (cpap) em recém-nascidos prematuros de extremo baixo peso. Revista da Universidade Vale do Rio Verde, Três Corações. 2013;11(2):209-17.

5. Coto VN, Molina ChR, Rivera SR. Intervención de enfermería obstétrica: un estudio de caso. Rev Enfermería Actual en Costa Rica [internet]. 2009 [citado 2014 jun 14];16. Disponible en: http://www.revenf.ucr.ac.cr/intervenc ionobstetrica.pdf

6. World Alliance for Patient Safety Forward Programme 2008-2009. Geneva: World Health Organization; 2010. Disponible en: http://www.scielo.org.mx/pdf/bmim/v69n5/v69n5a11.pdf

7. Organización Mundial de la Salud. Metas internacionales de seguridad del paciente. Disponible en: http:// www.who.int/mediacentre/news/

8. Ferrer MR, Cuesta GYL, Rodríguez FF, Estévez LMC. Supervivencia del recién nacido ventilado. AMC [internet]. 2012 [citado 2013 abr 21];16(2):190-8. Disponible en: http://scielo.sld.cu/pdf/amc/v16n2/amc080212.pdf

9. García FY, Fernández RRM, Rodríguez RM, Pérez ME. Supervivencia en el recién nacido ventilado. Rev Cubana Pediatr [internet]. 2006 dic [citado 2008 jul 7];78(4):[aprox. 3 p.]. Disponible en: http://www.scielo.sld.cu/ scielo.php?script $=$ sci_arttext $\&$ pid $=$

10. Montanholi LL, Merighi BMA, Pinto de JMC. Actuación de la enfermera en la unidad de terapia intensiva neonatal: entre lo ideal, lo real y lo posible. Rev Latino-Am Enfermagem [internet]. 2011 [citado 2013 jul 14];19(2): [08 pantallas]. Disponible en: http://www.scielo.br/pdf/rlae/v19n2/es_11.pdf

11. García H, Ramírez SJH, Ramírez FJ, Villegas SR, Madrigal MO. Frecuencia de lesión de la vía aérea identificada por broncoscopia en recién nacidos con intubación endotraqueal prolongada en una unidad de cuidados intensivos neonatales de tercer nivel. Bol Med Hosp Infant Mex [internet]. 2012 [citado 2013 nov 4];69(5):397-403. Disponible en: http://www.scielo.org.mx/pdf/bmim/v69n5/v69n5a11.pdf

12. Ley General de Salud en Materia de Investigación para la Salud México. 42 ed. México: Sista; 2013. Disponible en http://www.cndh.org.mx.../leyes20\%ynormnas $20 \%$

13. Aedo CV, Parada ST, Alcayaga RC, Rubio AM. Registro electrónico de enfermería en la valoración de las heridas. Enferm Glob [internet]. 2012 [citado 2013 abr 14];11(28): 386-95. Disponible en: http://revistas.um.es/eglo $\mathrm{bal} /$ article/viewle

14. Carballo PMC, Gómez ME. Características de las complicaciones pulmonares asociadas a la ventilación mecánica en recién nacidos. Pediatr. 2010;37(2):107-11. 
15. Navarro AJM. Revisión de los estudios sobre los profesionales de enfermería de las unidades de cuidados intensivos de España. Enferm Glob [internet]. 2012 [citado 2014 oct 16];11(26):267-89. Disponible en: http://scielo.isc iii.es/scielo.php

16. Duarte ED, Sena RR, Xavier CC. Processo de trabalho na unidade de terapia intensiva neonatal: construção de uma atenção orientada pela integralidade. Rev Esc Enferm USP [internet]. 2009 Sep [cited 2016 may 13];43(3):647-54. Disponible en: http://www.scielo.br/scielo.php?script=-6234200900030ng= http://dx.doi .org/10.1590/S0080-62342009000300021

17. Nascimento RM, Ferreira CAL, Coutinho ACFP y Verissimo CSSR. La frecuencia de lesión nasal en los recién nacidos, debido al uso de presión positiva continua de la vía aérea. Rev Latino-Am Enfermería [internet]. 2009 [citado 2014 abr 17];17(4):489-94. Disponible en: https://www.google.com.br/webhp?sourceid=chrome-a+c ontinua+\% $3 \% \mathrm{ADa}$

18. García RS, Navío MA, Valentin ML. Normas básicas para la elaboración de los registros de enfermería. Nure Invest. 2007;28:1-8.

19. González J, Gosgaya O, García M, Blesa A. Registros de enfermería: convencional frente a informatizado. Unidad de cuidados críticos. Enferm Intensiva. 2004;15(2):53-62.

20. Muñoz BJI. Ventilación mecánica en pediatría: conceptos generales. Ann Pediatr (Bar) [internet]. 2003 [citado 3 jul 2008];59(1):[aprox. 4 p.]. Disponible en: http://www.elsevier.es/revistas/ctl_servlet?_f $=7064 \& i p=201.22$ $0.215 .13 \&$ articuloid $=13048801 \&$ revistaid $=37$

21. Bulechek G, McCloskey DJ, Butcher H. Clasificación de intervenciones de enfermería (CIE) [internet]. 6a ed. Madrid: Mosby; 2014. Disponible en: http://es.slideshare.net/maryeliza16/clasificacin-de-intervenciones-de-e nfermera-nic-6ta-edicin-2014

22. Labarrere C, Castro LFW, González HG. Beneficio de la asistencia ventilatoria de alta frecuencia en el recién nacido. Rev Cubana Enfermer [Internet]. 2006 Jun [citado 3 jul 2014];22(2):[aprox. 12 p.]. Disponible en: http://ww w.scielo.sld.cu/scielo.php?script=sci_arttext\&pid=S0864-03192006000200004\&lng=es

23. Bancalari MA. Ventilación de alta frecuencia en recién nacidos: un soporte respiratorio necesario. Rev Chil Pediatr [internet]. 2003 [citado 3 jul 2013];74(5):[aprox. 11 p.]. Disponible en: http://www.scielo.cl/scielo.php?script $=$ sci_arttext\&pid $=$ S0370-41062003000500003\&lng=es\&nrm=iso\&tlng=es

24. Lara DVJ. Programa de actualización continua en neonatología (PAC) 3: libro 8 CPAP Neonatal. México: Federación Nacional de Neonatología de México; 2007.

25. Juárez RPA, García CML. La importancia del cuidado de enfermería. Rev Enferm. Instituto Mexicano del Seguro Social. 2009;17(2):113-5.

26. Muñoz SL, Luna FC. Modelo de cuidado: comunicación enfermera-neonato. ENE. Revista de Enfermería [internet]. 2012 [citado 2014 abr 30];6(3). Disponible en: http://ene-enfermeria.org/ojs/index.php/ENE/art icle/viewFile/210/186

27. Sousa NFC de, Bonfim S de FSF, Vasconcelos MGL de, Bezerra JL de O, Silva D VC da, Leal LP. Prevalência de lesão do septo nasal em prematuros no uso de pronas nasais. Rev Esc Enferm USP [internet]. 2013 Dic [citado 2014 may 11];47(6):1285-90. Disponible en: http://www.scielo.br/scielo.php?script=sci_arttext\&pid=S0080-

28. Montanholi LL, Merighi MAB, Jesus MCP. Actuación de la enfermera en la unidad de terapia intensiva neonatal: entre lo ideal, lo real y lo posible. Rev Latino-Am Enfermagem [internet]. 2011 Apr [citado 2016 may 16];19(2):301-8. Disponible en: http://www.scielo.br/scielo.php?script=sci_arttext\&pid=S0104-116920110 00200011\&lng=en. http://dx.doi.org/10.1590/S0104-

29. Bonfim SFSF, Vasconcelos MGL, Sousa NFC, Silva DVC, Leal LP. Lesión de tabique nasal en neonatos pretérmino en el uso de prongs nasales. Rev Latino-Am Enfermagem [internet]. 2014 oct [citado 2015 may 25];22(5):826-33. Disponible en: http://www.scielo.br/scielo.php?script=sci_arttext\&pid=S0104-11692014 000500826\&lng=en. http://dx.doi.org/10.1590/0104-1169.3451.2486 


\section{Notas}

* Artículo de investigación.

\section{BY}

Información adicional

Cómo citar: García Reza C, Mejía-Flores MA, Guadarrama Pérez L, Gómez Martínez V. Intervenciones de enfermería en neonatos con presión positiva continua. Investig Enferm. Imagen Desarr. 2018;20(1):xx-xx. h ttps://doi.org/10.11144/Javeriana.ie20-1.ienp 